\title{
On Comparison Study between Double Sumudu and Elzaki Linear Transforms Method for Solving Fractional Partial Differential Equations
}

\author{
Hasan Shather Kadhem ${ }^{1 *} \quad$ Sameer Qasim Hasan ${ }^{2}$
}

${ }^{1}$ Department of Mathematics, College of Education, Baghdad University, Baghdad, Iraq

${ }^{2}$ Department of Mathematics, College of Education, Al-Mustansiriyah University, Baghdad, Iraq

*Corresponding author: hasanshather1968@gmail.com, dr.sameerqasim@uomustansiriyah.edu.iq

*ORCID ID: https://orcid.org/0000-0001-9954-4777, https://orcid.org/0000-0002-2613-2584

Received 12/2/2019, Accepted 4/6/2020, Published Online First 21/2/2021, Published 1/9/2021

\begin{abstract}
:
In this paper, double Sumudu and double Elzaki transforms methods are used to compute the numerical solutions for some types of fractional order partial differential equations with constant coefficients and explaining the efficiently of the method by illustrating some numerical examples that are computed by using Mathcad 15.and graphic in Matlab R2015a.
\end{abstract}

Key words: Caputo derivatives, Fractional Calculus, Fractional Partial differential Equations, Single and double Sumudu, Elzaki transform.

\section{Introduction:}

Fractional differential equations are effective tools to formulate the physical problems. The oldest integral transform is Laplace transform by Laplace in $(1780)(1)$. Watugala introduced the Sumudu transform in (1993), which has some advantages over the Laplace transform $(2,3)$. The Elzaki transform, which was introduced by (4) in (2011), is a revised form of the two previous transforms. For more details and historical review of integral transform(5,6). In (7) double Laplace and double Sumudu transform were used to solve wave equation and Poisson equation. In this study double Sumudu transform and double Elzaki transform are considered to solve some fractional partial differential equations which conclude both the space and time or mixed fractional Caputo derivatives, such as parabolic- hyperbolic, wave and heat fractional equations.

\section{Preliminaries}

Now some of alimentary concepts of fractional calculus (6) that needed later on.

\section{Definition 1:(8,9)}

The R- L fractional integral of a function $f(t)$ with order $\tilde{\alpha}>0$ is defined as

$$
J^{\widetilde{\alpha}} f(t)=\frac{1}{\Gamma(\tilde{\alpha})} \int_{0}^{t}(t-x)^{\widetilde{\alpha}-1} f(x) d x \quad t>0 .
$$

The linear operator R-L fractional integral has the following properties:

$$
\begin{array}{ll}
\text { 1. } & J^{0}=I \\
\text { 2. } & J^{\widetilde{\alpha}} \cdot J^{\widetilde{\beta}}=J^{\widetilde{\beta}} \cdot J^{\widetilde{\alpha}}=J^{\widetilde{\alpha}+\widetilde{\beta}} \\
\text { 3. } & J^{\widetilde{\alpha}} t^{\widetilde{\beta}}=\frac{\Gamma(1+\widetilde{\beta})}{\Gamma(1+\widetilde{\beta}+\widetilde{\alpha}} t^{\widetilde{\alpha}+\widetilde{\beta}}> \\
0, \tilde{\beta}>-1, t>0 &
\end{array}
$$

The Caputo fractional derivative : $D_{t}^{\widetilde{\alpha}} f(t)=J^{n n-\widetilde{\alpha}} D_{t}^{n} f(t)$

$$
=\left\{\begin{array}{cc}
\frac{1}{\Gamma(n n-\tilde{\alpha})} \int_{0}^{t} \frac{f^{(n n)}(x)}{(t-x)^{n n-\tilde{\alpha}+1}} d x & n n-1<\tilde{\alpha}<n n \in N, \\
f^{(n n)}(x) & \tilde{\alpha}=n n \in N .
\end{array}\right.
$$

Some properties :

$$
\begin{aligned}
& \left\{\begin{array}{cc}
\frac{\Gamma(1+\widetilde{\beta})}{\Gamma(1+\widetilde{\beta}-\widetilde{\alpha})} t^{\widetilde{\alpha}} t^{\widetilde{\beta}}= & n-1<\tilde{\alpha}<n \quad \tilde{\beta}>n n-1, \quad \tilde{\beta} \in R, n \in N, \\
0 & n-1<\tilde{\alpha}<n, \quad \tilde{\beta} \leq n n-1, \quad \tilde{\beta}, n \in N
\end{array}\right. \\
& \begin{array}{l}
\text { 2. } \quad D_{t}^{\widetilde{\alpha}} J^{\widetilde{\alpha}}=I \\
\text { 3. } \quad J^{\widetilde{\alpha}}\left[D_{t}^{\widetilde{\alpha}} f(t)\right]=f(t)-\sum_{i=0}^{n-1} \frac{t^{i}}{i !} f^{(i)}(0)
\end{array} \\
& \text { 4. } \quad D_{t}^{\widetilde{\alpha}}\left[D_{t}^{\widetilde{\beta}} f(t)\right]=D_{t}^{\widetilde{\beta}}\left[D_{t}^{\widetilde{\alpha}} f(t)\right]=D_{t}^{\widetilde{\alpha}+\widetilde{\beta}} f(t) \\
& \text { provided that } \\
& f^{(i)}(0)=0, \quad i=0,1, \ldots, n n-1, \alpha+\beta \leq n n, \\
& \quad n n \in N
\end{aligned}
$$

The most important function in fractional calculus is the Mittag-Leffler function which is a generalized for the exponent function and is defined by the following definition 


\section{Definition 2:(10)}

The Mittag-Leffler function with two parameters is defined as:

$$
\begin{aligned}
E_{\widetilde{\alpha}, \widetilde{\beta}}(x)=\sum_{k=0}^{\infty} \frac{x^{k}}{\Gamma(\tilde{\alpha} k+\tilde{\beta})} & \tilde{\alpha}, \tilde{\beta} \in C, \\
\operatorname{Rea}(\tilde{\alpha}), & \operatorname{Rea}(\tilde{\beta})>0
\end{aligned}
$$

\section{Lemma 1:(11)}

The Mittag-Leffler function has interesting properties (11):

1. $E_{\widetilde{\alpha}, 1}(x)=E_{\widetilde{\alpha}}(x)$

2. $E_{1,1}(x)=e^{x}, \quad x E_{1,2}(x)=e^{x}-1$

3. $E_{2,1}\left(x^{2}\right)=\cosh (x)$

4. $x E_{2,1}\left(x^{2}\right)=\sinh (x)$

5. $D_{t}^{\widetilde{\gamma}}\left(t^{\widetilde{\beta}-1} E_{\widetilde{\alpha}, \widetilde{\beta}}\left(a t^{\widetilde{\alpha}}\right)\right)=$ $t^{\widetilde{\beta}-\widetilde{\gamma}-1} E_{\widetilde{\alpha}, \widetilde{\beta}-\widetilde{\gamma}}\left(a t^{\widetilde{\alpha}}\right) \quad \tilde{\gamma}>0$

6. $E_{2,1}\left(-x^{2}\right)=\cos (x)$

7. $x E_{2,1}\left(-x^{2}\right)=\sin (x)$

8. $E_{\widetilde{\alpha}, \widetilde{\beta}}^{(n)}(x)=\sum_{k=0}^{\infty} \frac{(k+n) ! x^{k}}{k ! \Gamma(\widetilde{\alpha} k+\widetilde{\alpha} n+\widetilde{\beta})} \quad n \in N$

9. $D_{t}^{\widetilde{\alpha}}\left(E_{\widetilde{\alpha}}\left(a t^{\widetilde{\alpha}}\right)\right)=a E_{\widetilde{\alpha}}\left(a t^{\widetilde{\alpha}}\right) \quad \tilde{\alpha}>0, \quad a \in R$

In order to prove the results, the following definitions and theorems will be need.

Definition 3: (2) A function $f(x)$ is said to be of exponent order $\alpha>0$ if there exist non-negative constants $M, \alpha$ and $T$ such that $|f(x)| \leq$ $M e^{\alpha x} \quad x \geq T$.

Definition 4 (3) The Sumudu transform of the exponent order function $f(t)$ is defined as

$$
\begin{gathered}
\hat{S}[f(t)]=\int_{0}^{\infty} f(p t) e^{-t} d p=\int_{0}^{\infty} \frac{1}{p} f(t) e^{-\frac{t}{p}} d u \\
=\hat{\mathcal{T}}(p), \quad t, p>0
\end{gathered}
$$

Table 1 contains Sumudu transform for some

\begin{tabular}{|c|c|c|c|}
\hline$f(x)$ & $\begin{array}{l}S[f(x)] \\
=\mathcal{T}[p]\end{array}$ & $f(x)$ & $\begin{array}{l}S[f(x)] \\
=\mathcal{T}[p]\end{array}$ \\
\hline 1 & 1 & $\boldsymbol{x}^{\widetilde{\alpha}}$ & $\boldsymbol{\Gamma}(\mathbf{1}+\tilde{a p}) \boldsymbol{p}^{\tilde{\alpha}}$ \\
\hline$e^{x}$ & $\overline{1-a p}$ & $\sin a x$ & $\overline{1+a^{2} p^{2}}$ \\
\hline $\cos a x$ & $\frac{1}{1+a^{2} p^{2}}$ & $\sinh a x$ & $\frac{a p}{1-a^{2} p^{2}}$ \\
\hline$x^{\breve{\beta}-1} E_{\widetilde{\alpha}, \breve{\beta}}\left(\mu x^{\breve{\beta}}\right)$ & $\frac{p^{\beta-1}}{1-\mu p^{\check{\alpha}}}$ & $\cosh a x$ & $\frac{1}{1-a^{2} p^{2}}$ \\
\hline
\end{tabular}
famous function.

Table 1. Sumudu transform of some important functions
Interesting conditions for existing the inverse of Sumudu transform are concluded in the next Theorem.

Theorem 1: (2) Let $\widehat{\mathcal{T}}(p)$ be the Sumudu transform of the function $f(t)$ and

1. $\frac{\hat{T}\left(\frac{1}{s}\right)}{s}$ is a meromorphic function, $\operatorname{Re}(s)<$

2. $\left|\frac{\hat{T}(1 / s)}{s}\right|<b r^{-a}, a, b>0$. Then $f(t)=$

$$
\begin{aligned}
& \hat{S}^{-1}\left[\hat{\mathcal{T}}(p]=\frac{1}{2 \pi i} \int_{a-i \infty}^{a+i \infty} e^{s t} \frac{\hat{\mathcal{T}}\left(\frac{1}{s}\right)}{s} d s=\right. \\
& \sum_{\text {Residual }}\left[e^{s t} \frac{\hat{\mathcal{T}}\left(\frac{1}{s}\right)}{s}\right]
\end{aligned}
$$

Lemma 2: (2) Sumudu transformation of fractional integral for $f(t)$ is given by

$$
\hat{S}\left[I^{\widetilde{\alpha}} f(t)\right]=p^{\widetilde{\alpha}} \widehat{\mathcal{T}}(p)
$$

Provided definition of fractional Caputo derivatives, some of important properties of the Sumudu transformation are listed here.

\section{Some properties of Sumudu transform (2)}

1. $\hat{S}\left[f^{(n)}(t)\right]=$

$$
p^{-n}\left[\hat{\mathcal{T}}(p)-\sum_{k=0}^{n} p^{-k} f^{(k)}(0)\right]
$$

2. $\hat{S}\left[D^{\widetilde{\alpha}} f(t)\right]=$

$$
p^{-\widetilde{\alpha}}\left[\widehat{\mathcal{T}}(p)-\sum_{k=0}^{n} p^{-k} f^{(k)}(0)\right]
$$

3. $\hat{S}\left\{t^{\widetilde{\beta}-1} E_{\widetilde{\alpha}, \widetilde{\beta}}(\lambda t)\right\}=\frac{p^{\tilde{\beta}-1}}{1-\lambda p^{\widetilde{\alpha}}}$

Definition 5: (3) The double Sumudu transform of the two variable function $f(x, t) ; x, t \in R^{+}$is defined by :

$$
\begin{array}{r}
\hat{\mathcal{T}}[p, q]=\hat{S}_{2}[f(x, t)] \\
=\frac{1}{p q} \int_{0}^{\infty} \int_{0}^{\infty} e^{-\left(\frac{t}{q}+\frac{x}{p}\right)} f(x, t) d x d t
\end{array}
$$

Theorem 2: (2) Let $\frac{\partial^{i+j} f(x, t)}{\partial t^{j} \partial x^{i}}, \quad i=0,1, \ldots, n ; \quad j=0,1,2, \ldots, m$ be of exponent order, then

$$
\begin{array}{ll}
\text { 1. } & \hat{S}_{2}\left[\frac{\partial^{n} f(x, t)}{\partial x^{n}}\right]= \\
& p^{-n}\left[\hat{\mathcal{T}}[p, q]-\sum_{i=0}^{n-1} p \hat{\mathcal{T}}_{i}[0, q]\right] \\
\text { 2. } & \hat{S}_{2}\left[\frac{\partial^{m} f(x, t)}{\partial t^{m}}\right]=q^{-m}[\mathcal{T}[p, q]- \\
& \left.\sum_{j=0}^{m-1} q^{j} \hat{\mathcal{T}}_{i}[p, 0]\right] \\
\text { 3. } & \hat{S}_{2}\left[\frac{\partial^{m+n} f(x, t)}{\partial t^{m} \partial x^{n}}\right]=q^{-m} p^{-n}[\hat{\mathcal{T}}[p, q]- \\
& \sum_{i=0}^{n-1} p^{i} \widehat{\mathcal{T}}_{i}[0, q]-\sum_{j=0}^{m-1} q^{j} \mathcal{T}_{i}[p, 0]+ \\
& \left.\sum_{j=0}^{m-1} \sum_{i=0}^{n-1} p^{i} q^{j} \frac{\partial^{i+j}}{\partial t^{j} x^{i}} f(0,0)\right]
\end{array}
$$

Where $\hat{\mathcal{T}}_{i}[0, q]=\hat{S}_{2}\left[\frac{\partial^{i}}{\partial x^{i}} f(0, t)\right], \widehat{\mathcal{T}}_{i}[p, 0]=$ $\hat{S}_{2}\left[\frac{\partial^{j}}{\partial t^{j}} f(x, 0)\right]$. 
For the two variables exponent order function $(x, t)$, the double Sumudu transform of the partial fractional integrals are given by the following theorem

Theorem 3: (2) If $f(x) \in L_{1}((0, a) \times$ $(0, b)), a, b>0$, then

$\hat{S}_{2}\left[I_{x}^{\widetilde{\alpha}} f(x, t)\right], \hat{S}_{2}\left[I_{t}^{\widetilde{\beta}} f(x, t)\right], \hat{S}_{2}\left[I_{t}^{\widetilde{\beta}} I_{x}^{\widetilde{\alpha}} f(x, t)\right]$ exist and

$$
\text { 1. } \hat{S}_{2}\left[I_{x}^{\widetilde{\alpha}} f(x, t)\right]=p^{\widetilde{\alpha}} \hat{\mathcal{T}}(p, q)
$$$$
\text { 2. } \hat{S}_{2}\left[I_{t}^{\widetilde{\beta}} f(x, t)\right]=q^{\widetilde{\beta}} \widehat{\mathcal{T}}(p, q)
$$$$
\text { 3. } \hat{S}_{2}\left[I_{t}^{\widetilde{\beta}} I_{x}^{\alpha} f(x, t)\right]=p^{\widetilde{\alpha}} q \widehat{\mathcal{T}}^{\widetilde{\beta}} \mathcal{T}(p, q)
$$

Theorem 4: (2) Let $f(x, t)$ be a function with exponent order and has continuous partial derivatives on $R^{+} \times R^{+}$and these derivatives are in $L_{1}((0, a) \times(0, b))$ then

$$
\begin{array}{ll}
\text { 1. } & \hat{S}_{2}\left[D_{x}^{\widetilde{\alpha}} f(x, t)\right]=p^{-\widetilde{\alpha}}[\widehat{\mathcal{T}}(p, q)- \\
& \left.\sum_{i=0}^{n-1} q^{i} \widehat{\mathcal{T}}_{i}(0, q)\right] \\
\text { 2. } & \hat{S}_{2}\left[D_{t}^{\widetilde{\beta}} f(x, t)\right]=q[\widehat{\mathcal{T}}(p, q)- \\
& \left.\sum_{j=0}^{m-1} q^{j} \hat{\mathcal{T}}_{j}(p, 0)\right]
\end{array}
$$

3. $\hat{S}_{2}\left[D_{t}^{\widetilde{\beta}} D_{x}^{\alpha} f(x, t)\right]=p^{-\alpha}[\hat{\mathcal{T}}(p, q)-$

$$
\begin{aligned}
& \sum_{i=0}^{n-1} p^{i} \widehat{\mathcal{T}}_{i}(0, q)-\sum_{j=0}^{m-1} q^{j} \widehat{\mathcal{J}}_{j}(p, 0)+ \\
& \left.\sum_{j=0}^{m-1} \sum_{i=0}^{n-1} p^{i} q^{j} \frac{\partial^{i+j}}{\partial t^{j} x^{i}} f(0,0)\right]
\end{aligned}
$$

\section{Lemma 3:}

Assuming that $\quad n-1<\tilde{\alpha}<n n, m m-1<$ $\tilde{\beta}<m m, \tilde{\alpha}, \tilde{\beta}>0, m m, n \in N$, then

$$
\begin{aligned}
\hat{S}_{2}\left[D_{t}^{\widetilde{\beta}} D_{t}^{\widetilde{\alpha}} f(x, t)\right] & q^{-\widetilde{\beta}}\left[q^{-\widetilde{\alpha}}(\widehat{\mathcal{T}}(p, q)\right. \\
& \left.-\sum_{j=0}^{n-1} q^{j} \widehat{\mathcal{J}}_{j}(p, 0)\right) \\
& \left.-\sum_{j=0}^{m-1} q^{j} \hat{S}_{2}\left\{D_{t}^{j} D_{t}^{\widetilde{\alpha}} f(x, 0)\right\}\right]
\end{aligned}
$$

Proof:

According to Theorem (5), and using properties of fractional integral and Caputo derivatives, will get:

$$
\begin{aligned}
& \hat{S}_{2}\left[D_{t}^{\widetilde{\beta}} D_{t}^{\widetilde{\alpha}} f(x, t)\right]=\hat{S}_{2}\left[I^{m m-\widetilde{\beta}} D_{t}^{m} D_{t}^{\widetilde{\alpha}} f(x, t)\right] \\
& =q^{m m-\widetilde{\beta}} \hat{S}_{2}\left[D_{t}^{m m}\left(D_{t}^{\widetilde{\alpha}} f(x, t)\right)\right] \\
& =q^{m m-\widetilde{\beta}}\left[q ^ { - m m } \left[\hat{S}_{2}\left[D_{t}^{\widetilde{\alpha}} f(x, t)\right]\right.\right. \\
& \left.-\sum_{j=0}^{m-1} q^{j} \hat{S}_{2}\left[D_{t}^{j} D_{t}^{\widetilde{\alpha}} f(x, 0)\right]\right] \\
& =q^{-\widetilde{\beta}}\left[q^{-\widetilde{\alpha}}\left(\hat{\mathcal{T}}(p, q)-\sum_{j=0}^{n-1} q^{j} \widehat{\mathcal{T}}_{j}(p, 0)\right)\right. \\
& \left.-\sum_{j=0}^{m m-1} q \hat{S}_{2}\left[D_{t}^{j} D_{t}^{\widetilde{\alpha}} f(x, 0)\right]\right]
\end{aligned}
$$

Theorem 5: (12) If $\widehat{\mathcal{T}}[p, q]$ is the double Sumudu transform of the two variables function $f(x, t)$, then

$\hat{S}_{2}\left\{x^{m} t^{n} f(x, t)\right\}=$

$p^{m} q^{n} \sum_{k=0}^{m} \sum_{l=0}^{n} a_{k}^{m} b_{l}^{n} p^{k} q^{l} \frac{\partial^{k+l}}{\partial p^{k} \partial q^{l}} \hat{\mathcal{T}}[p, q]$, where

$a_{0}^{m}=1, \quad a_{m}^{m}=1, \quad a_{1}^{m}=m m !, \quad a_{m-1}^{m}=m^{2}$

similarly for $b_{l}^{n}$ for any $k=2,3, \ldots, n-2$

Furthermore, $a_{k}^{m}=a_{k-1}^{m-1}+(m+k) a_{k}^{m-1}$

similarly for $b_{l}^{n}$

\section{Elzaki Transform}

A modification to Sumudu transform is a new transform 'Elzaki transform' which is introduced by Tarig Elzaki in 2010, for solving a class of ordinary differential equations with variable coefficients under special initial and boundary conditions, that cannot be done by the Sumudu transform or Laplace transform only (13). Thus, Elzaki transform is a more effective than the Sumudu transform. The researcher (3) designed some conditions for solving such class of differential equations by Elzaki transform method only, these conditions convert the origin equation into other with constant coefficients which can be easy to solve.

But in case of linear ordinary differential equations with constant coefficients, Elzaki and Sumudu transforms usually give the same solution through the duality between the two transforms.

Definition 5 :(3) Elzaki transform of the exponent order function $f(t)$ is defined by

$$
\begin{aligned}
\tilde{E}[f(t)]=p^{2} \int_{0}^{\infty} & e^{-t} f(p t) d t \quad 0 r \\
& =p \int_{0}^{\infty} e^{-\frac{t}{p}} f(t) d t=\mathcal{G}(p), \quad p \\
& >0
\end{aligned}
$$


If $\tilde{E}[f(t)]=\mathcal{G}(p)$, then (14):

1. $\quad \mathcal{G}(p)=p^{2} \hat{\mathcal{T}}(p)$ duality with Sumudu transform.

2. $\tilde{E}\left[f^{(n)}(t)\right]=p^{-n}\left[\mathcal{G}(p)-\sum_{k=0}^{n-1} p f^{(k)}(0)\right]$.

3. $\tilde{E}[t f(t)]=p^{2} \frac{d}{d p} \mathcal{G}(p)-p \cdot \mathcal{G}(p)$

4. $\quad \tilde{E}\left[t^{2} f(t)\right]=p^{4} \frac{d^{2}}{d p^{2}} \mathcal{G}(p)$

5. $\quad \tilde{E}\left[t^{3} f(t)\right]=p^{6} \frac{d^{3}}{d p^{3}} \mathcal{G}(p)+3 p^{5} \frac{d^{2}}{d p^{2}} \mathcal{G}(p)$

6. $\tilde{E}[t f(t)]=p^{2} \frac{d}{d p}\left[\frac{\mathcal{G}(p)}{p}-f(0)\right]-$ $p\left[\frac{\mathcal{G}(p)}{p}-f(0)\right]$

7. $\tilde{E}\left[t^{2} f(t)\right]=p^{4} \frac{d^{2}}{d p^{2}}\left[\frac{G(p)}{p}-f(0)\right]$

Theorem 6: (14) If $\tilde{E}\left[f^{(n)}(t)\right]=\mathcal{G}_{n}(p)$, then:

1. $\tilde{E}\left[t f^{(n)}(t)\right]=p^{2} \frac{d}{d p} \mathcal{G}_{n}(p)-p \mathcal{G}_{n}(p)$

2. $\tilde{E}\left[t^{2} f^{(n)}(t)\right]=p^{4} \frac{d^{2}}{d p^{2}} \mathcal{G}_{n}(p)$

\section{Definition 6 :(14)}

Let the function $f(x, t)$ be an exponent order, and $x, t>0$, then the double Elzaki transform for $f$ is given by

$$
\begin{gathered}
\tilde{E}_{2}[f(x, t)]=p q \int_{0}^{\infty} \int_{0}^{\infty} f(x, t) e^{-\left(\frac{x}{p}+\frac{t}{q}\right)} d x d t \\
=\mathcal{G}(p, q)
\end{gathered}
$$

Whenever the improper integral is convergent.

The duality of double Elzaki transform with double Sumudu transform is similar for that single case, i.e $\mathcal{G}(p, q)=p^{2} q^{2} \hat{\mathcal{T}}(p, q)$.

\section{Some illustrative Examples}

The first example is to explain the advantage of the Elzaki transform over the Sumudu transform, through the following simple linear fractional ordinary differential equation with variable coefficients which is generalized for that in (15).

Example 1: Consider the following variable coefficients fractional ordinary differential equation

$$
\begin{aligned}
a t x^{\widetilde{\boldsymbol{\alpha}}}+b x=c t, & 0<\widetilde{\boldsymbol{\alpha}} \leq 1, \\
\text { condition } & x(0)=d
\end{aligned}
$$

with initial

\section{Solution by Sumudu Transform Method}

Operating Sumudu transform for both sides of (1) and using Theorem (6) to get

$$
\begin{gathered}
a S\left[t x^{\widetilde{\alpha}}\right]+b S[x]=c S[t] \\
a v^{2} \frac{d}{d v}\left[\frac{\hat{\mathcal{T}}(v)-x(0)}{v^{\tilde{\alpha}}}\right]+a v\left[\frac{\hat{\mathcal{T}}(v)-x(0)}{v^{\tilde{\alpha}}}\right]+b \hat{\mathcal{T}}(v)=c v,
\end{gathered}
$$$$
\text { where } \hat{\mathcal{T}}(v)=S[x(t)]
$$

Substitute the initial condition and simplify to get

$$
\begin{aligned}
& \mathrm{av}^{2} \frac{\mathrm{d}}{\mathrm{dv}}\left[\frac{\widehat{\mathcal{T}}(\mathrm{v})}{\mathrm{v}^{\widetilde{\alpha}}}\right]-\mathrm{adv}^{2} \frac{\mathrm{d}}{\mathrm{dv}}\left[\frac{1}{\mathrm{v}^{\widetilde{\alpha}}}\right]+\mathrm{av}^{1-\widetilde{\alpha}} \widehat{\mathcal{T}}(\mathrm{v}) \\
& -a d v^{1-\widetilde{\alpha}}+\mathrm{b} \hat{\mathcal{T}}(\mathrm{v})=\mathrm{cv}
\end{aligned}
$$

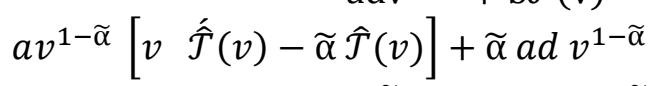

$$
\begin{aligned}
& +a v^{1-\widetilde{\alpha}} \widehat{\mathcal{T}}(v)-a d v^{1-\widetilde{\alpha}}+b \hat{\mathcal{T}}(v) \\
& =c v \\
& a v^{2-\widetilde{\alpha}} \hat{\mathcal{T}}(v)-a \alpha v^{1-\widetilde{\alpha}} \hat{\mathcal{T}}(v)-\widetilde{\alpha} a d v^{1-\widetilde{\alpha}} \\
& +a v^{1-\widetilde{\alpha}} \widehat{\mathcal{T}}(v)-a d v^{1-\widetilde{\alpha}}+b \hat{\mathcal{T}}(v) \\
& =c v \\
& \hat{\mathcal{T}}(v)+\left[\frac{b}{a v^{2-\widetilde{\boldsymbol{\alpha}}}}-\frac{\widetilde{\boldsymbol{\alpha}}}{v}+\frac{1}{v}\right] \widehat{\mathcal{T}}(v)=\frac{c}{a v^{1-\widetilde{\boldsymbol{\alpha}}}}-\frac{\widetilde{\boldsymbol{\alpha}} d}{v}+\frac{d}{v}
\end{aligned}
$$

Then for all $\widetilde{\boldsymbol{\alpha}} \in(0,1]$, again this equation is the first order ordinary fractional differential equation with variable coefficients, so cannot solve it by Sumudu transform method only.

\section{Solution by Elzaki Transform Method}

Operating Elzaki transform for both sides of (1) and using Theorem (7) to get

$$
\begin{gathered}
a E\left[t x^{\widetilde{\alpha}}\right]+b E[x]=c E[t] \\
a v^{2} \frac{d}{d v}\left[\frac{\mathcal{G}(v)-v^{2} x(0)}{v^{\widetilde{\alpha}}}\right]-a v\left[\frac{\mathcal{G}(v)-v^{2} x(0)}{v^{\widetilde{\alpha}}}\right] \\
+b \mathcal{G}(v)=c v^{3} \\
a v^{1-\widetilde{\boldsymbol{\alpha}}}[v \dot{\mathcal{G}}(v)-\widetilde{\boldsymbol{\alpha}} \mathcal{G}(v)]-(2-\alpha) v^{3-\widetilde{\boldsymbol{\alpha}}} d \\
-a v^{1-\widetilde{\boldsymbol{\alpha}}} \mathcal{G}(v)+a d v^{3-\widetilde{\boldsymbol{\alpha}}}+b \mathcal{G}(v) \\
=c v^{3} \\
\dot{\mathcal{G}}(v)-\left[\frac{1}{v}+\frac{\widetilde{\boldsymbol{\alpha}}}{v}-\frac{b}{\left.a v^{2-\widetilde{\boldsymbol{\alpha}}}\right]}\right] \mathcal{G}(v) \\
-\frac{(2-\widetilde{\boldsymbol{\alpha}}) v^{3-\alpha} d-a d v^{3-\alpha}}{a v^{2-\widetilde{\boldsymbol{\alpha}}}} \\
=\frac{c v^{3}}{a v^{2-\widetilde{\boldsymbol{\alpha}}}}
\end{gathered}
$$

With stander case $\widetilde{\boldsymbol{\alpha}}=1$, if the initial condition changed to $=0$, then will get

$\dot{\mathcal{G}}(v)-\left[\frac{2 a-b}{a v}\right] \mathcal{G}(v)=\frac{c v^{2}}{a}$.

So, by letting $b=2 a$, for example ' $a=1$ '

The last equation is converted to $\dot{\mathcal{G}}(v)=c v^{2}$ which has the solution $\mathcal{G}(v)=\frac{c v^{3}}{3}=v^{3}$ with $c=3$, taking inverse Elzaki transform, will have

$$
x(t)=t
$$

Other cases $\alpha \neq 1$, in order to get an equation with constant coefficients, coefficient of $G(v)$ must be

vanish, i. e $\quad\left[\frac{1}{v}+\frac{\widetilde{\alpha}}{v}-\frac{b}{a v^{2-\widetilde{\alpha}}}\right]=0 \Rightarrow$ $a(1+a) v^{1-\widetilde{\boldsymbol{\alpha}}}=b$. So, Elzaki transform alone failed to solve this fractional ordinary differential equation with variable coefficients.

Now some of fractional partial differential equations with Caputo derivatives are given to solve it by both double Sumudu and Elzaki transforms methods to get an exact solution for these equations. 
In the following example, will generalize example 1 in (16) to fractional order time derivatives with new conditions.

Example 2: Consider the following:

$$
\frac{\partial^{\tilde{\beta}}}{\partial t^{\tilde{\beta}}} f+\frac{\partial^{\tilde{\tilde{\alpha}}}}{\partial t^{\widetilde{\alpha}}} f+f=f_{x x}, \quad 1<\widetilde{\beta} \leq
$$

$2, \quad 0.5<\widetilde{\alpha} \leq 1$

With the conditions

$$
\begin{aligned}
& f(x, 0)=0, \quad f_{t}(x, 0)=e^{x}, f(0, t) \\
& =t E_{\widetilde{\beta}-\widetilde{\alpha}, 2}\left(-t^{\widetilde{\beta}-\widetilde{\alpha}}\right)=f_{x}(0, t)
\end{aligned}
$$

\section{Solution by Double Sumudu Transform}

Single Sumudu transform for the conditions yields:

$$
\begin{array}{cl}
\widehat{\mathcal{T}}_{0}[p, 0]=0, & \hat{\mathcal{T}}_{1}[p, 0]=\frac{1}{1-p}, \quad \widehat{\mathcal{T}}_{0}[0, q] \\
= & \widehat{\mathcal{T}}_{1}[0, q]=\frac{q}{1+q^{\widetilde{\beta}-\widetilde{\alpha}}}
\end{array}
$$

Now, taking double Sumudu transform for the equation (2), to get:

$$
\hat{S}_{2}\left(\frac{\partial^{\widetilde{\beta}}}{\partial t^{\widetilde{\beta}}} f\right)+\hat{S}_{2}\left(\frac{\partial^{\widetilde{\widetilde{\alpha}}}}{\partial t^{\widetilde{\widetilde{\alpha}}}} f\right)+\hat{S}_{2}(f)=\hat{S}_{2}\left(f_{x x}\right)
$$

Using Sumudu transform properties through Theorem (4) yeilds

$$
\begin{aligned}
& q^{-\widetilde{\beta}}\left[\widehat{\mathcal{T}}[p, q]-\widehat{\mathcal{T}}_{0}[p, 0]-q \widehat{\mathcal{T}}_{1}[p, 0]\right]+ \\
& q^{-\widetilde{\alpha}}\left[\hat{\mathcal{T}}[p, q]-\widehat{\mathcal{T}}_{0}[p, 0]\right]+\hat{\mathcal{T}}[p, q]= \\
& p^{-2}\left[\hat{\mathcal{T}}[p, q]-\widehat{\mathcal{T}}_{0}[0, q]-p \widehat{\mathcal{T}}_{1}[0, q]\right] \\
& q^{-\widetilde{\beta}}\left(\widehat{\mathcal{T}}[p, q]-\frac{q}{1-p}\right)+q^{-\widetilde{\alpha}} \hat{\mathcal{T}}[p, q]+\hat{\mathcal{T}}[p, q] \\
& =p^{-2}\left(\widehat{\mathcal{T}}[p, q]-\frac{q}{1+q^{\widetilde{\beta}-\widetilde{\alpha}}}\right. \\
& \left.-\frac{p q}{1+q^{\widetilde{\beta}-\widetilde{\alpha}}}\right) \\
& p^{2} q^{\widetilde{\alpha}}\left(\widehat{\mathcal{T}}[p, q]-\frac{q}{1-p}\right)+p^{2} q^{\widetilde{\alpha}+\widetilde{\beta}} \hat{\mathcal{T}}[p, q] \\
& =q^{\widetilde{\alpha}+\widetilde{\beta}}\left(\widehat{\mathcal{T}}[p, q]-\frac{(1+p) q}{1+q^{\widetilde{\beta}-\widetilde{\alpha}}}\right) \\
& \left(p^{2} q^{\widetilde{\alpha}}+p^{2} q^{\widetilde{\beta}}+p^{2} q^{\widetilde{\beta}+\widetilde{\alpha}}-q^{\widetilde{\alpha}+\widetilde{\beta}}\right) \hat{\mathcal{T}}[p, q] \\
& =\frac{q\left(p^{2} q^{\widetilde{\alpha}}+p^{2} q^{\widetilde{\beta}}+p^{2} q^{\widetilde{\beta}+\widetilde{\alpha}}-q^{\widetilde{\alpha}+\widetilde{\beta}}\right)}{(1-p)\left(1+q^{\widetilde{\beta}-\widetilde{\alpha}}\right)} \\
& \hat{\mathcal{T}}[p, q]=\left(\frac{1}{1-p}\right)\left(\frac{q}{1+q^{\widetilde{\beta}-\widetilde{\alpha}}}\right)
\end{aligned}
$$

Applying the inverse double Sumudu transformation provided with Lemma 1 and properties of the Sumudu transform, to get $f(x, t)=e^{x} t E_{\widetilde{\beta}-\widetilde{\alpha}, 2}\left(-t^{\widetilde{\beta}-\widetilde{\alpha}}\right)$
For $\tilde{\alpha}=1, \tilde{\beta}=2$, then the standard equation (2) has the exact solution as $f(x, t)=e^{x}\left(1-e^{-t}\right)$

Which is the same as in (16).

\section{Solution bv Double Elzaki Transform}

Single Elzaki transform for the conditions yields:

$$
\begin{gathered}
\mathcal{G}_{0}[p, 0]=0, \quad \mathcal{G}_{1}[p, 0]=\frac{\mathrm{p}^{2}}{1-\mathrm{p}}, \quad \mathcal{G}_{0}[0, \mathrm{q}] \\
=\frac{\mathrm{q}^{3}}{1+\mathrm{q}^{\beta-\alpha}}=\mathcal{G}_{1}[0, \mathrm{q}]
\end{gathered}
$$

Now, taking double Elzaki transform for the equation (2), then get:

$$
\begin{aligned}
& q^{-\widetilde{\beta}}\left[\mathcal{G}[p, q]-q^{2} \mathcal{G}_{0}[p, 0]-q^{3} \mathcal{G}_{1}[p, 0]\right] \\
& +q^{-\widetilde{\alpha}}\left[\mathcal{G}[p, q]-p^{2} \mathcal{G}_{0}[p, 0]\right] \\
& +\mathcal{G}[p, q] \\
& =p^{-2}\left[\mathcal{G}[p, q]-p^{2} \mathcal{G}_{0}[p, 0]\right. \\
& \left.-p^{3} \mathcal{G}_{1}[0, \mathrm{q}]\right] \\
& q^{-\widetilde{\beta}}\left(\mathcal{G}[p, q]-\frac{q^{3} p^{2}}{1-p}\right)+q^{-\widetilde{\alpha}} \mathcal{G}[p, q]+\mathcal{G}[p, q] \\
& =p^{-2}\left(\mathcal{G}[p, q]-\frac{q^{3} p^{2}}{1+q^{\widetilde{\alpha}+\widetilde{\beta}}}\right. \\
& \left.-\frac{q^{3} p^{3}}{1+q^{\widetilde{\alpha}+\widetilde{\beta}}}\right) \\
& p^{2} q^{\widetilde{\alpha}}\left(\mathcal{G}[p, q]-\frac{q^{3} p^{2}}{1-p}\right)+p^{2} q^{\widetilde{\alpha}+\widetilde{\beta}} \mathcal{G}[p, q] \\
& =q^{\widetilde{\alpha}+\widetilde{\beta}}\left(\mathcal{G}[p, q]-\frac{q^{3} p^{2}(1+p)}{1+q^{\widetilde{\alpha}+\widetilde{\beta}}}\right) \\
& \left(p^{2} q^{\widetilde{\alpha}}+p^{2} q^{\widetilde{\beta}}+p^{2} q^{\widetilde{\alpha}+\widetilde{\beta}}-q^{\widetilde{\alpha}+\widetilde{\beta}}\right) \mathcal{G}[p, q] \\
& =\frac{q^{3} p^{2}\left(p^{2} q^{\widetilde{\alpha}}+p^{2} q^{\widetilde{\beta}}+p^{2} q^{\widetilde{\alpha}+\widetilde{\beta}}-q^{\widetilde{\alpha}+\widetilde{\beta}}\right)}{(1-u)\left(1+q^{\alpha+\beta}\right)} \\
& \mathcal{G}[p, q]=\frac{p^{2}}{1-p} \frac{q^{3}}{1+q^{\widetilde{\alpha}+\widetilde{\beta}}}
\end{aligned}
$$

And by applying the inverse double Elzaki transformation, one can obtain the exact solution as:

$$
f(x, t)=e^{x} t E_{\widetilde{\beta}-\widetilde{\alpha}, 2}\left(-t^{\widetilde{\beta}-\widetilde{\alpha}}\right)
$$

Standard equation (2) has the exact solution when $\tilde{\alpha}=1, \tilde{\beta}=2$ as

$$
f(x, t)=e^{x}\left(1-e^{-t}\right)
$$

That agrees with that for double Sumudu transform method.

The absolute error of some of 10 -order approximate solutions for equation (2) for different values of $\tilde{\alpha}, \tilde{\beta}$, are included in Table 2. Fig. 1 illustrates the solution. 
Table 2. Absolute error of 10-order approximate solutions for equation (2)

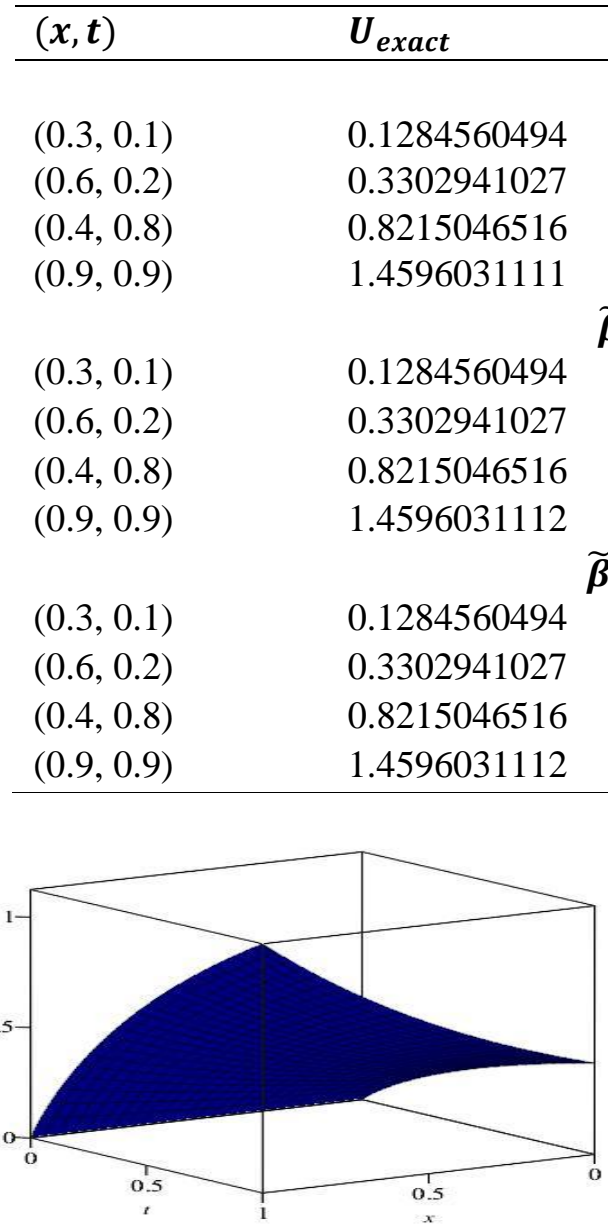

(a) $\operatorname{Exact} \tilde{\beta}=2, \tilde{\alpha}=1$

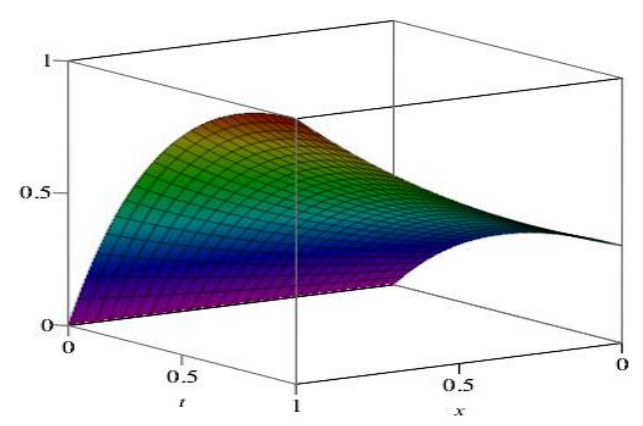

(c) $\tilde{\beta}-\tilde{\alpha}=0.75$

\begin{tabular}{cl}
\multicolumn{1}{c}{$\boldsymbol{U}_{\mathbf{1 0} \text { app. }}$} & $\mid$ Error $\mid$ \\
$\widetilde{\boldsymbol{\beta}}-\widetilde{\boldsymbol{\alpha}}=\mathbf{1}$ & \\
0.1284560494 & $0.9999999998 \mathrm{e}-20$ \\
0.3302940982 & $1.5350000000 \mathrm{e}-17$ \\
0.8215046518 & $2.0156684416 \mathrm{e}-10$ \\
1.4596031125 & $1.3559212604 \mathrm{e}-9$ \\
$\widetilde{\boldsymbol{\beta}}-\widetilde{\boldsymbol{\alpha}}=\mathbf{0 . 9}$ & \\
0.126125092 & $2.3309574273 \mathrm{e}-03$ \\
0.3215691316 & $8.7249711979 \mathrm{e}-03$ \\
0.7943540994 & $2.7150552172 \mathrm{e}-02$ \\
1.4145467839 & $4.5056327265 \mathrm{e}-02$ \\
$\widetilde{\boldsymbol{\beta}}-\widetilde{\boldsymbol{\alpha}}=\mathbf{0 . 7 5}$ & \\
0.1212593423 & $7.1967071225 \mathrm{e}-03$ \\
0.305402764 & $2.4891338769 \mathrm{e}-02$ \\
0.7556966072 & $6.5808044384 \mathrm{e}-02$ \\
1.3519946908 & $1.0760842039 \mathrm{e}-01$
\end{tabular}

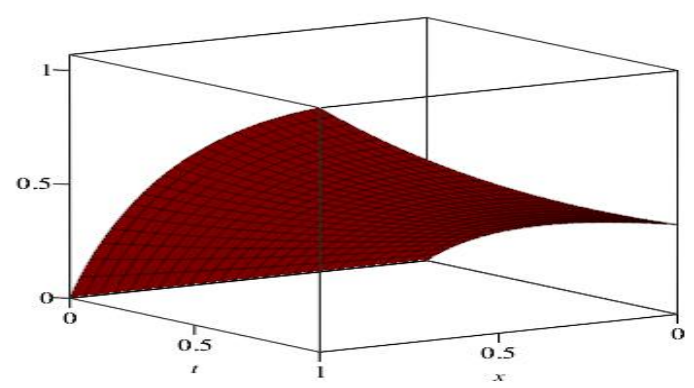

(b) approx. $\tilde{\beta}-\tilde{\alpha}=1$

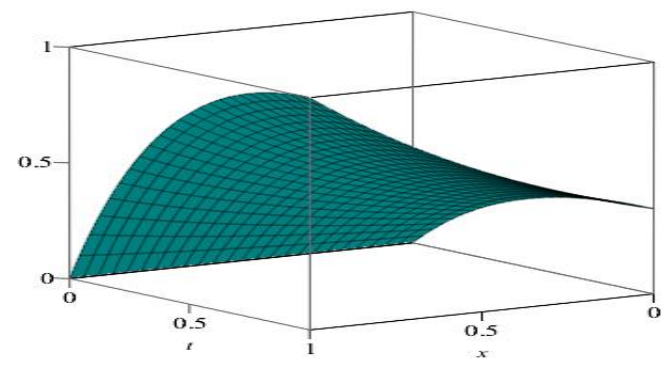

(d) $\tilde{\beta}-\tilde{\alpha}=0.6$

Figure 1. Exact solution and 10-order approximate solutions of Equation (2) for different values of $\tilde{\boldsymbol{\alpha}, \tilde{\boldsymbol{\beta}} \text {. }}$

Example 3: Consider the following fractional space-time telegraph equation:

$$
\begin{gathered}
\frac{\partial^{\widetilde{\beta}}}{\partial t^{\widetilde{\beta}}} f+\frac{\partial^{\widetilde{\alpha}}}{\partial t^{\widetilde{\alpha}}} f+f=\frac{\partial^{\tilde{\gamma}}}{\partial x^{\widetilde{\gamma}}} f, \quad 1<\tilde{\beta}, \tilde{\gamma} \leq 2, \\
0.5<\tilde{\alpha} \leq 1
\end{gathered}
$$

Subject to the initial and boundary conditions

$$
\begin{array}{r}
f(x, 0)=E_{\widetilde{\gamma}}\left(x^{\widetilde{\gamma}}\right)+x E_{\widetilde{\gamma}, 2}\left(x^{\widetilde{\gamma}}\right), \quad f_{t}(x, 0) \\
=-\left(E_{\widetilde{\gamma}}\left(x^{\widetilde{\gamma}}\right)+x E_{\widetilde{\gamma}, 2}\left(x^{\widetilde{\gamma}}\right)\right)
\end{array}
$$

$$
f(0, t)=1-t E_{\widetilde{\beta}-\widetilde{\alpha}, 2}\left(-t^{\widetilde{\beta}-\widetilde{\alpha}}\right)=f_{x}(0, t)
$$

Solution by Double Sumudu Transform

By applying the single Sumudu transform for the conditions, and using Mittag Leffler function properties, to have 


$$
\begin{aligned}
\hat{\mathcal{T}}_{0}[p, 0]=\frac{1+p}{1-p^{\widetilde{\gamma}}}, \quad \widehat{\mathcal{T}}_{1}[p, 0] & \\
= & \frac{-(1+p)}{1-p^{\widetilde{\gamma}}}, \quad \widehat{\mathcal{T}}_{0}[0, q] \\
& =1-\frac{q}{1+q^{\widetilde{\beta}-\widetilde{\alpha}}}
\end{aligned}
$$

Therefore as above

$$
\begin{aligned}
& q^{-\widetilde{\beta}}\left(\widehat{\mathcal{T}}[p, q]-\widehat{\mathcal{T}}_{0}[p, 0]-q \widehat{\mathcal{T}}_{1}[p, 0]\right) \\
& +q^{-\widetilde{\alpha}}\left(\widehat{\mathcal{T}}[p, q]-\widehat{\mathcal{T}}_{0}[p, 0]\right) \\
& +\hat{\mathcal{T}}[p, q] \\
& =p^{-\widetilde{\gamma}}\left(\hat{\mathcal{T}}[p, q]-\widehat{\mathcal{T}}_{0}[0, q]\right. \\
& \left.-p \widehat{\mathcal{T}}_{1}[0, q]\right) \\
& q^{-\widetilde{\alpha}}\left(\hat{\mathcal{T}}[p, q]-\frac{(1+p)(1-q)}{1-p^{\widetilde{\gamma}}}\right) \\
& +q^{-\widetilde{\alpha}}\left(\widehat{\mathcal{T}}[p, q]-\frac{1+p}{1-p^{\widetilde{\gamma}}}\right) \\
& +\widehat{\mathcal{T}}[p, q] \\
& =p^{-\widetilde{\gamma}}(\hat{\mathcal{T}}[p, q]-(1+p)(1 \\
& \left.\left.-\frac{q}{1+q^{\widetilde{\beta}-\widetilde{\alpha}}}\right)\right) \\
& \left(p^{\widetilde{\gamma}} \tilde{\alpha}+p^{\widetilde{\gamma}} q^{\widetilde{\beta}}+p^{\widetilde{\gamma}} q^{\widetilde{\alpha}+\widetilde{\beta}}-q^{\widetilde{\alpha}+\widetilde{\beta}}\right) T(p, q) \\
& =\frac{p^{\widetilde{\gamma}} q^{\widetilde{\alpha}}(1+p)(1-q)}{1-p^{\widetilde{\gamma}}} \\
& +\frac{p^{\widetilde{\gamma}} q^{\widetilde{\beta}}(1+p)}{1-p^{\widetilde{\gamma}}}-q^{\widetilde{\alpha}+\widetilde{\beta}}(1+p)(1 \\
& \left.-\frac{q}{1+q^{\widetilde{\beta}-\widetilde{\alpha}}}\right) \\
& =(1+p)\left[\frac{p^{\widetilde{\gamma}} q^{\widetilde{\alpha}}(1-q)+p^{\widetilde{\gamma}} q^{\widetilde{\beta}}}{1-p^{\widetilde{\gamma}}}-q^{\widetilde{\alpha}+\widetilde{\beta}}(1\right. \\
& \left.\left.-\frac{q}{1+q^{\widetilde{\beta}-\widetilde{\alpha}}}\right)\right]
\end{aligned}
$$

By simplify,

$$
\widehat{\mathcal{T}}[p, q]=\frac{(1+p)\left(1-q+q^{\widetilde{\beta}-\widetilde{\alpha}}\right)}{\left(1-p^{\widetilde{\gamma}}\right)\left(1+q^{\widetilde{\beta}-\widetilde{\alpha}}\right)}
$$

And by applying the inverse double Sumudu transformation one can obtain the exact solution as:

$$
\begin{array}{r}
f(x, t)=\left[E_{\widetilde{\gamma}}\left(x^{\widetilde{\gamma}}\right)+x E_{\widetilde{\gamma}, 2}\left(x^{\widetilde{\gamma}}\right)\right][1 \\
\left.-t E_{\widetilde{\beta}-\widetilde{\alpha}, 2}\left(-t^{\widetilde{\beta}-\widetilde{\alpha}}\right)\right]
\end{array}
$$

When $\tilde{\alpha}=1, \tilde{\beta}=\tilde{\gamma}=2$, then have the standard telegraph equation (3) which has $f(x, t)=e^{x-t}$ as an exact solution.

Table 3 contain the absolute error for some approximated solutions for equation (2) and some of solutions with different values of $\tilde{\alpha}, \tilde{\beta}$ and $\tilde{\gamma}$ are illustrated graphically in Fig. 2

\section{Solution bv Double Elzaki Transform}

By applying the single Elzaki transform for the conditions, and using Mittag-Leffler function properties

$$
\begin{aligned}
\mathcal{G}_{0}[p, 0]=\frac{p^{2}(1+p)}{1-p^{\widetilde{\gamma}}}, \quad \mathcal{G}_{1}[p, 0] & \\
= & \frac{-p^{2}(1+p)}{1-p^{\widetilde{\gamma}}}, \quad \mathcal{G}_{0}[0, \mathrm{q}] \\
& =q^{2}-\frac{q^{3}}{1+q^{\widetilde{\beta}-\widetilde{\alpha}}}=\mathcal{G}_{1}[0, \mathrm{q}]
\end{aligned}
$$

Operating double Elzaki transform and using Lemma(2), gives

$$
\begin{aligned}
& q^{-\beta}\left(\mathcal{G}[p, q]-p^{2} \mathcal{G}_{0}[p, 0]-q^{3} \mathcal{G}_{1}[p, 0]\right) \\
& +q^{-\widetilde{\alpha}}\left(\mathcal{G}[p, q]-q^{2} \mathcal{G}_{0}[p, 0]\right) \\
& +\mathcal{G}[p, q] \\
& =p^{-\widetilde{\gamma}}\left(\mathcal{G}[p, q]-p^{2} \mathcal{G}_{0}[0, \mathrm{q}]\right. \\
& \left.-p^{3} \mathcal{G}_{1}[0, \mathrm{q}]\right) \\
& q^{-\widetilde{\beta}}\left(\mathcal{G}[p, q]-\frac{q^{2} p^{2}(1+p)(1-q)}{1-p^{\widetilde{r}}}\right) \\
& +q^{-\widetilde{\alpha}}\left(\mathcal{G}[p, q]-\frac{q^{2} p^{2}(1+q)}{1-q^{\widetilde{\gamma}}}\right) \\
& +\mathcal{G}[p, q] \\
& =p^{-\widetilde{\gamma}}\left(\mathcal{G}[p, q]-p^{2}(1+p)\left(q^{2}\right.\right. \\
& \left.\left.-\frac{q^{3}}{1+q^{\widetilde{\beta}-\widetilde{\alpha}}}\right)\right) \\
& \left(p^{\widetilde{\gamma}} q^{\widetilde{\alpha}}+p^{\widetilde{\gamma}} q^{\widetilde{\beta}}+p^{\widetilde{\gamma}} q^{\widetilde{\alpha}+\widetilde{\beta}}-q^{\widetilde{\alpha}+\widetilde{\beta}}\right) \mathcal{G}[p, q] \\
& =p^{2} q^{2}\left[\frac{q^{\widetilde{\alpha}} p^{\widetilde{\gamma}}(1+p)(1-q)}{1-p^{\widetilde{\gamma}}}\right. \\
& +\frac{q^{\widetilde{\beta}} p^{\widetilde{\gamma}}(1+p)}{1-p^{\widetilde{\gamma}}}-q^{\widetilde{\alpha}+\widetilde{\beta}}(1+p)(1 \\
& \left.\left.-\frac{q}{1+q^{\widetilde{\beta}-\widetilde{\alpha}}}\right)\right] \\
& =p^{2} q^{2}(1+p)\left[\frac{q^{\widetilde{\alpha}} p^{\widetilde{\gamma}}(1-q)+q^{\widetilde{\beta}} p^{\widetilde{\gamma}}}{1-p^{\widetilde{\gamma}}}-q^{\widetilde{\alpha}+\widetilde{\beta}}(1\right. \\
& \left.\left.-\frac{q}{1+q^{\widetilde{\beta}-\widetilde{\alpha}}}\right)\right]
\end{aligned}
$$

By simplifying

$$
\begin{aligned}
\mathcal{G}[p, q]=\frac{p^{2} q^{2}(1+p)\left(1-q+q^{\widetilde{\beta}-\widetilde{\alpha}}\right)}{\left(1-p^{\widetilde{\gamma}}\right)\left(1+q^{\widetilde{\beta}-\widetilde{\alpha}}\right)} \\
=\frac{p^{2}(1+p)}{\left(1-p^{\widetilde{\gamma}}\right)} \frac{q^{2}\left(1-q+q^{\widetilde{\beta}-\widetilde{\alpha}}\right)}{\left(1+q^{\widetilde{\beta}-\widetilde{\alpha}}\right)}
\end{aligned}
$$

So, by applying the inverse double Elzaki transform, to get the exact solution of equation (2) as

$$
\begin{array}{r}
f(x, t)=\left[E_{\widetilde{\gamma}}\left(x^{\widetilde{\gamma}}\right)+x E_{\widetilde{\gamma}, 2}\left(x^{\widetilde{\gamma}}\right)\right][1 \\
\left.-t E_{\widetilde{\beta}-\widetilde{\alpha}, 2}\left(-t^{\widetilde{\beta}-\widetilde{\alpha}}\right)\right]
\end{array}
$$


The exact solution of equation(2) is $f(x, t)=e^{x-t}$ when $\tilde{\alpha}=1, \tilde{\beta}=\tilde{\gamma}=2$.

That agrees with that for Sumudu transform method.
Fig. 2 contains3D-plotted of some numerical solutions of Eq. (3) with different fractional orders $\tilde{\beta}-\tilde{\alpha}$ and $\tilde{\gamma}$.

Also, Table 3 shows the results of absolutely error for some 10- order approximate solutions.

Table 3. Absolutely error for some 10-order approximate solutions of Equation(3)

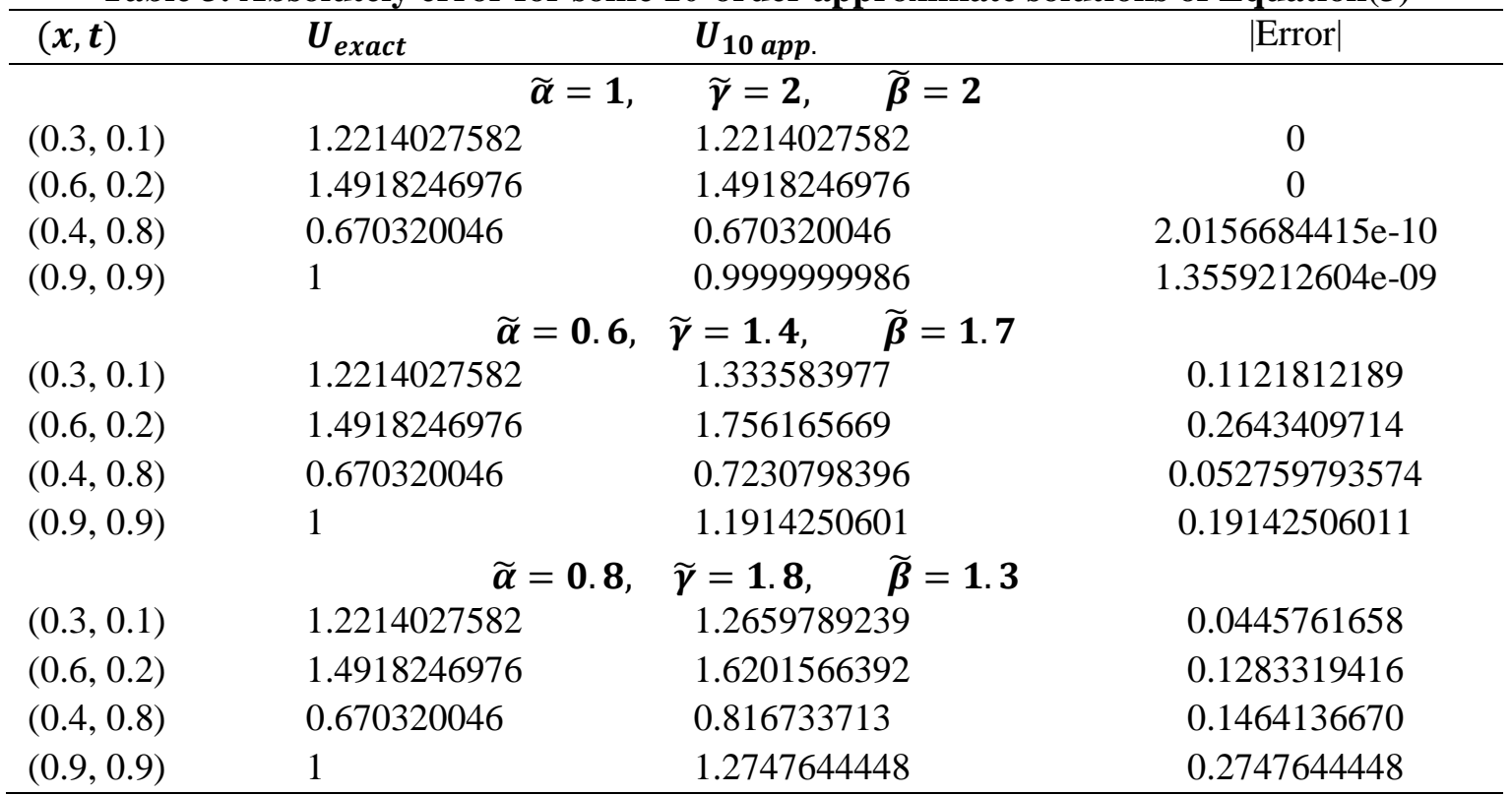
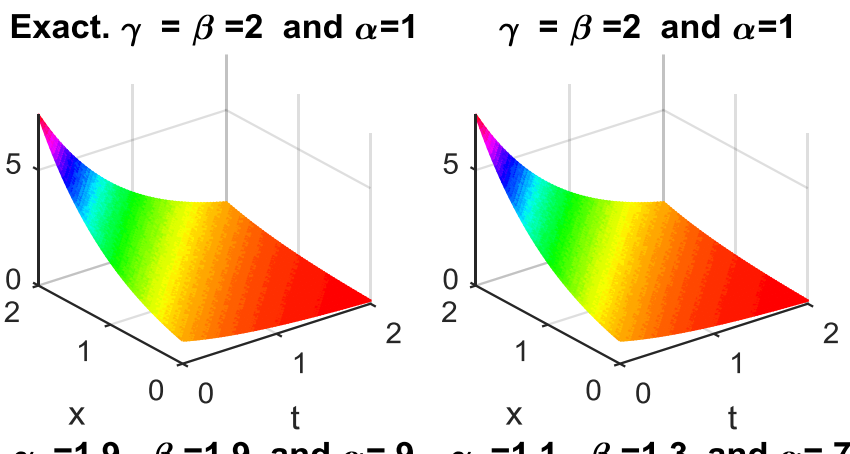

$\gamma=1.8, \beta=1.3$ and $\alpha=.8$
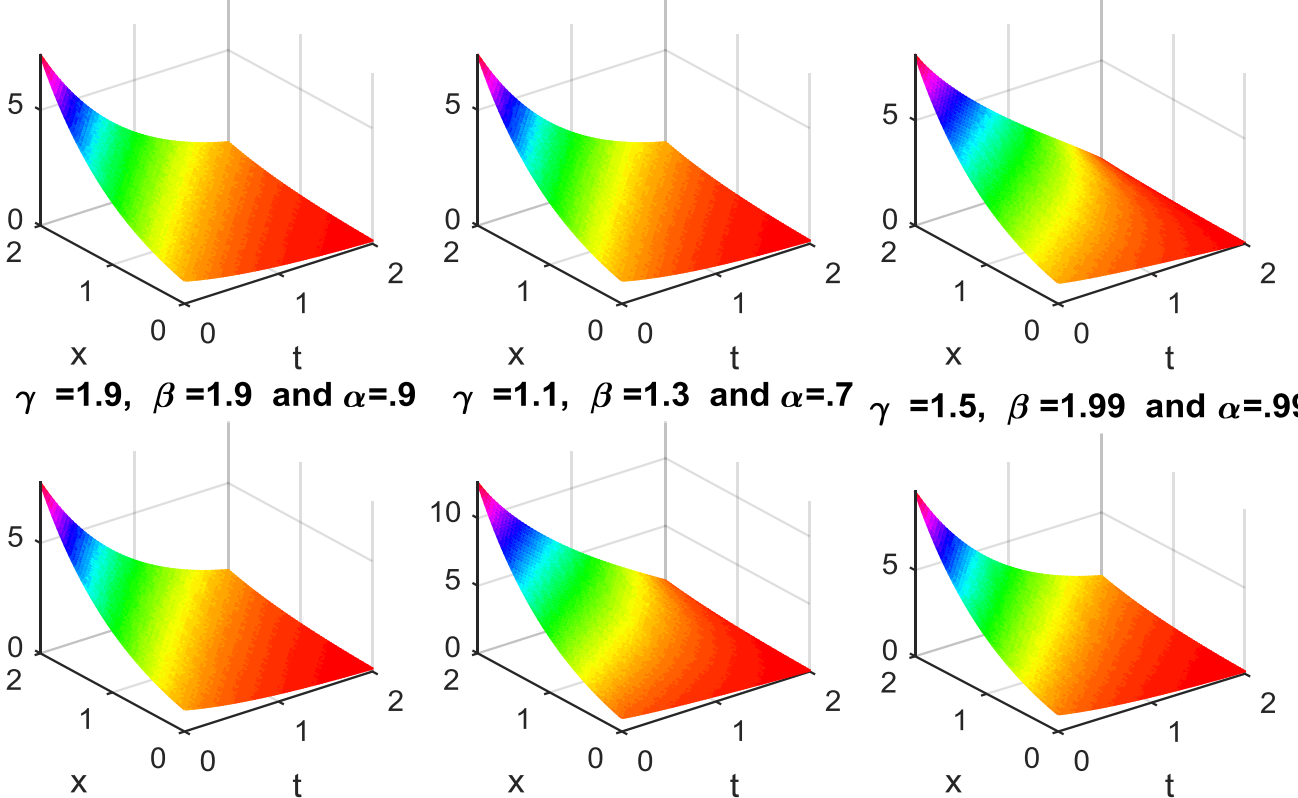

Figure 2. 10-order approximate solutions and the exact solution of Equation (3) for different values of $\widetilde{\alpha}, \widetilde{\beta}$ and $\widetilde{\gamma}$.

Next example is a generalized to space and time fractional order for that in ( 14 )

Example 4: Consider the following inhomogeneous fractional wave equation

$$
\left(\frac{\partial^{\widetilde{\alpha}}}{\partial t^{\partial}}-\frac{\partial^{\widetilde{\beta}}}{\partial x^{\widetilde{\beta}}}\right) f(x, t)=6 t+2 x, \quad 1
$$

With conditions

$$
<\tilde{\alpha}, \tilde{\beta} \leq 2
$$




$$
\begin{gathered}
f(x, 0)=0, \quad f_{t}(x, 0)=x E_{\widetilde{\beta}, 2}\left(-x^{\widetilde{\beta}}\right), \quad f(0, t) \\
=\frac{6 t^{1+\widetilde{\alpha}}}{\Gamma(\widetilde{\alpha}+2)}, \quad f_{x}(0, t) \\
=\frac{2 t^{\widetilde{\alpha}}}{\Gamma(1+\tilde{\alpha})}+t E_{\widetilde{\alpha}, 2}\left(-t^{\widetilde{\alpha}}\right)
\end{gathered}
$$

\section{Solution bv Double Sumudu Transform}

Applying the single Sumudu transform for the conditions

$$
\begin{array}{ll}
\hat{\mathcal{T}}_{0}[p, 0]=0, & \hat{\mathcal{T}}_{1}[p, 0]=\frac{p}{1+p^{\widetilde{\beta}}}, \\
& \hat{\mathcal{T}}_{0}[0, q]=6 q^{1+\widetilde{\alpha}}, \hat{\mathcal{T}}_{1}[0, q] \\
& =2 q^{\widetilde{\alpha}}+\frac{q}{1+q^{\widetilde{\alpha}}}
\end{array}
$$

By operating double Sumudu transform for both sides of equation (4) and simplifying the results, produce

$$
\begin{aligned}
& q^{-\widetilde{\alpha}}\left(\hat{\mathcal{T}}[p, q]-\frac{p q}{1+p^{\widetilde{\beta}}}\right) \\
& =p^{-\widetilde{\beta}}\left(\widehat{\mathcal{T}}[p, q]-6 q^{1+\widetilde{\alpha}}-p\left(2 q^{\widetilde{\alpha}}\right.\right. \\
& \left.\left.+\frac{q}{1+q^{\widetilde{\alpha}}}\right)\right)+6 q+2 p \\
& \left(p^{\widetilde{\beta}}-q^{\widetilde{\alpha}}\right) \hat{\mathcal{T}}[p, q] \\
& =\frac{p^{1+\widetilde{\beta}} q}{1+p^{\widetilde{\beta}}} \\
& -q^{\widetilde{\alpha}}\left(6 q^{1+\widetilde{\alpha}}+2 p q^{\widetilde{\alpha}}+\frac{p q}{1+q^{\widetilde{\alpha}}}\right) \\
& +2 p^{\widetilde{\beta}} q^{\widetilde{\alpha}}(p+3 q) \\
& =\frac{p q\left(p^{\widetilde{\beta}}-q^{\widetilde{\alpha}}\right)}{\left(1+p^{\widetilde{\beta}}\right)\left(1+q^{\widetilde{\alpha}}\right)}+2 q^{\widetilde{\alpha}}(p+3 q)\left(p^{\widetilde{\beta}}-q^{\widetilde{\alpha}}\right) \\
& \therefore \hat{\mathcal{T}}[p, q]=\frac{p}{1+p^{\widetilde{\beta}}} \frac{q}{1+q^{\widetilde{\alpha}}}+2 q^{\widetilde{\alpha}}(p+3 q)
\end{aligned}
$$

Applying the inverse double Sumudu transformation, one can obtain the exact solution for equation (4) as:

$$
\begin{aligned}
f(x, t)=x E_{\widetilde{\beta}, 2}\left(-x^{\widetilde{\beta}}\right) t E_{\widetilde{\alpha}, 2}\left(-t^{\widetilde{\alpha}}\right)+\frac{2 x t^{\widetilde{\alpha}}}{\Gamma(\tilde{\alpha}+1)} \\
+\frac{6 t^{1+\widetilde{\alpha}}}{\Gamma(\widetilde{\alpha}+2)}
\end{aligned}
$$

Standard wave equation (4) has $\quad f(x, t)=x t^{2}+$ $t^{3}+\sin (x) \sin (t)$ as an exact solution when $\tilde{\alpha}=\widetilde{\beta}=2$, which agree with that for (14).

\section{Solution bv Double Elzaki Transform}

Operating single Elzaki transform for our conditions

$$
\begin{aligned}
\mathcal{G}_{0}[p, 0]=0, \quad & \mathcal{G}_{1}[p, 0]=\frac{p^{3}}{1+p^{\widetilde{\beta}}}, \quad \mathcal{G}_{0}[0, \mathrm{q}] \\
= & 6 q^{\widetilde{\alpha}+3}, \quad \mathcal{G}_{1}[0, \mathrm{q}] \\
= & 2 q^{\widetilde{\alpha}+2}+\frac{q^{3}}{1+q^{\widetilde{\alpha}}}
\end{aligned}
$$

Double Elzaki transform for both sides of equation (3) yields

$$
\begin{gathered}
q^{-\widetilde{\alpha}}\left(\mathcal{G}[p, q]-\frac{p^{3} q^{3}}{1+p^{\widetilde{\beta}}}\right) \\
=p^{-\widetilde{\beta}}\left[\mathcal{G}[p, q]-6 q^{\widetilde{\alpha}+3} p^{2}\right. \\
\left.-p^{3}\left(2 q^{\widetilde{\alpha}+2}+\frac{q^{3}}{1+q^{\widetilde{\alpha}}}\right)\right] \\
+2 p^{2} q^{2}(p+3 q) \\
\left(p^{\widetilde{\beta}}-q^{\widetilde{\alpha}}\right) \mathcal{G}[p, q] \\
=p^{2} q^{2}\left[\frac{p^{1+\widetilde{\beta}} q}{1+p^{\widetilde{\beta}}}\right. \\
-q^{\widetilde{\alpha}}\left(6 q^{\widetilde{\alpha}+1}+2 p q^{\widetilde{\alpha}}+\frac{p q}{1+q^{\widetilde{\alpha}}}\right) \\
\left.+2 p^{\widetilde{\beta}} q^{\widetilde{\alpha}}(p+3 q)\right] \\
p^{2} q^{2}\left[\frac{p q\left(q^{\widetilde{\beta}}-q^{\widetilde{\alpha}}\right)}{\left(1+p^{\widetilde{\beta}}\right)\left(1+q^{\widetilde{\alpha}}\right)}+2 q^{\widetilde{\alpha}}(p\right. \\
\mathcal{G}[p, q]=\frac{p^{3}}{1+p^{\widetilde{\beta}}} \frac{q^{3}}{1+q^{\widetilde{\alpha}}+2 q^{\widetilde{\alpha}+2} p^{3}+6 q^{\widetilde{\alpha}+3} p^{2}}
\end{gathered}
$$

And by applying the inverse double Elzaki transformation, one can obtain the exact solution for equation

as:

$$
\begin{aligned}
& f(x, t)=x E_{\widetilde{\beta}, 2}\left(-x^{\widetilde{\beta}}\right) t E_{\widetilde{\alpha}, 2}\left(-t^{\widetilde{\alpha}}\right)+\frac{2 x t^{\widetilde{\alpha}}}{\Gamma(\widetilde{\alpha}+1)}+ \\
& \frac{6 t^{1+\widetilde{\alpha}}}{\Gamma(\widetilde{\alpha}+2)}
\end{aligned}
$$

Which agrees with that for double Sumudu transform method.

Fig. 3 contains3D-plotted of some numerical solutions of Eq. (3) with different fractional orders $\tilde{\beta}-\tilde{\alpha}$ and $\tilde{\gamma}$.

Also, Table 4 shows the results of absolutely error for some 10- order approximate solutions. 
Table 4. Exact and 10-order approximate solutions of Equation (4) for various values of $\tilde{\alpha}, \tilde{\beta}$.

\begin{tabular}{lccl}
\hline$(\boldsymbol{x}, \boldsymbol{t})$ & $\boldsymbol{U}_{\text {exact }}$ & \multicolumn{1}{c}{$\boldsymbol{U}_{\mathbf{1 0} \text { app. }}$} & $\mid$ Error $\mid$ \\
\hline & \multicolumn{2}{c}{$\widetilde{\boldsymbol{\alpha}}=\widetilde{\boldsymbol{\beta}}=\mathbf{2}$} \\
$(0.3,0.1)$ & 0.0335027919 & 0.0335027919 & 0 \\
$(0.6,0.2)$ & 0.1441771423 & 0.1441771423 & 0 \\
$(0.4,0.8)$ & 1.0473516198 & 1.0473516198 & 0 \\
$(0.9,0.9)$ & 2.0716010473 & 2.0716010473 & 0 \\
& $\widetilde{\boldsymbol{\alpha}}=\mathbf{1 . 6}$, & $\boldsymbol{\beta}=\mathbf{1 . 9 5}$ & \\
$(0.3,0.1)$ & 0.0335027919 & 0.0438920618 & 0.0103892699 \\
$(0.6,0.2)$ & 0.1441771423 & 0.1985157432 & 0.0543386009 \\
$(0.4,0.8)$ & 1.0473516198 & 1.5517716698 & 0.5044200500 \\
$(0.9,0.9)$ & 2.0716010473 & 2.8451114067 & 0.7735103593 \\
& $\widetilde{\boldsymbol{\alpha}}=\mathbf{1 . 7}$, & $\widetilde{\boldsymbol{\beta}}=\mathbf{1 . 3}$ & \\
$(0.3,0.1)$ & 0.0335027919 & 0.0421679509 & 0.0086651590 \\
$(0.6,0.2)$ & 0.1441771423 & 0.185687181 & 0.0415100387 \\
$(0.4,0.8)$ & 1.0473516198 & 1.5313726976 & 0.4840210779 \\
$(0.9,0.9)$ & 2.0716010473 & 2.7576361375 & 0.6860350901 \\
\hline
\end{tabular}

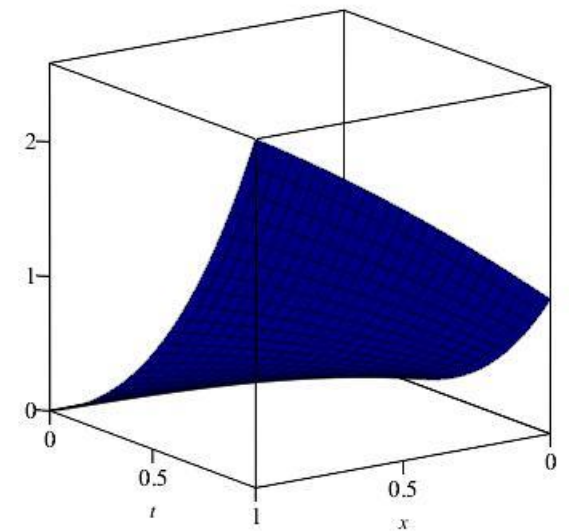

(a) Exact $\tilde{\alpha}=\tilde{\beta}=2$

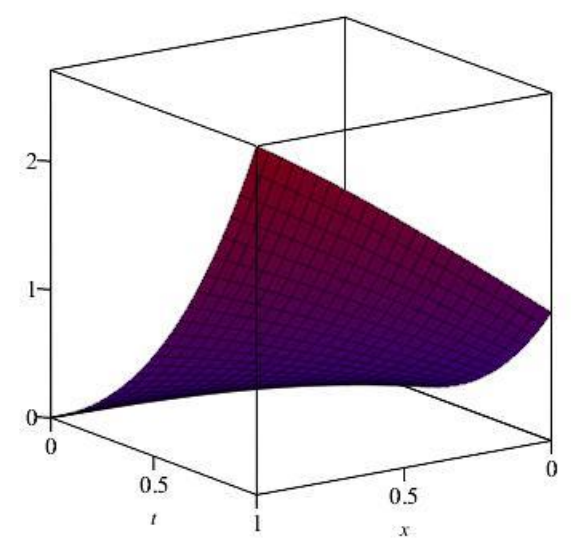

(c) $\widetilde{\boldsymbol{\beta}}=1.85, \widetilde{\boldsymbol{\alpha}}=1.5$

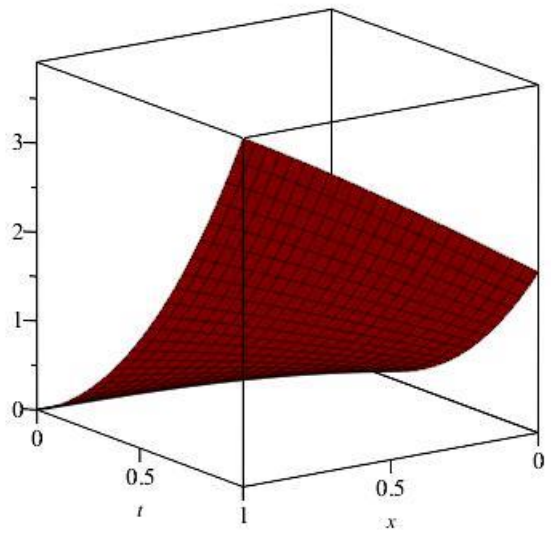

(b) Approx. $\widetilde{\boldsymbol{\alpha}}=\widetilde{\boldsymbol{\beta}}=\mathbf{2}$

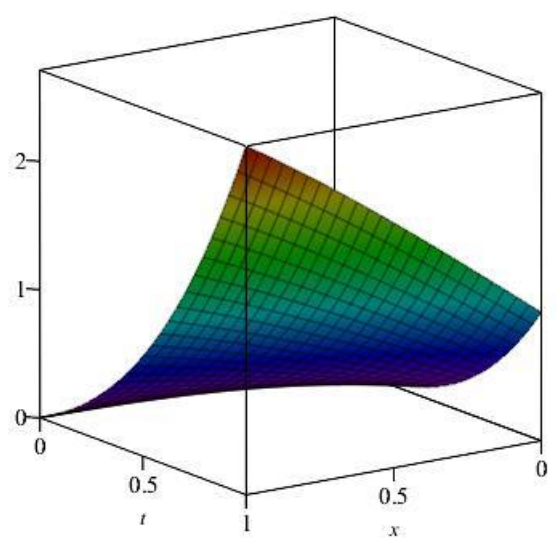

(d) $\widetilde{\boldsymbol{\beta}}=1.3, \widetilde{\boldsymbol{\alpha}}=2$

Figure 3. Exact solutions and some of 10-order approximate solutions of equation (4), with various values of $\widetilde{\boldsymbol{\beta}}, \widetilde{\boldsymbol{\alpha}}$. 
Example 4: Consider the nonhomogeneous parabolic-hyperbolic fractional partial differential equation

$$
\begin{aligned}
\left(\frac{\partial^{\widetilde{\alpha}}}{\partial t^{\widetilde{\alpha}}}-\frac{\partial^{\widetilde{\beta}}}{\partial x^{\widetilde{\beta}}}\right)\left(\frac{\partial^{\widetilde{\beta}}}{\partial t^{\widetilde{\beta}}}-\frac{\partial^{\widetilde{\beta}}}{\partial x^{\widetilde{\beta}}}\right) f(x, t) & =\Gamma(\tilde{\alpha}+\tilde{\beta}+1)\left(x^{\widetilde{\beta}}-t^{\widetilde{\beta}}\right. \\
& \left.-\frac{\Gamma(1+\tilde{\beta})}{\Gamma(1+\tilde{\alpha})} t^{\widetilde{\alpha}}\right), \\
& 0<\tilde{\alpha} \leq 1,1<\tilde{\beta} \leq 2
\end{aligned}
$$

With conditions

$$
\begin{gathered}
f(0,0)=0, \quad f_{x}(0, t)=f_{x x x}(0, t)=0, \quad f_{x x}(0, t) \\
=t^{\alpha+\beta}, \quad f_{t}(x, 0)=0
\end{gathered}
$$

\section{Solution bv Double Sumudu Transform.}

Single Sumudu transform of the conditions yields

$$
\begin{gathered}
\widehat{\mathcal{T}}(0,0)=0, \quad \widehat{\mathcal{T}}_{1}[0, q]=\widehat{\mathcal{T}}_{3}[0, q]=0, \quad \widehat{\mathcal{T}}_{2}[0, q] \\
=\Gamma(\tilde{\alpha}+\widetilde{\beta}+1) q^{\widetilde{\alpha}+\widetilde{\beta}} \\
\widehat{\mathcal{T}}_{1}[p, 0]=0
\end{gathered}
$$

From Theorem (6), and Lemma (2)

$\hat{S}_{2}\left[D_{t}^{\widetilde{\alpha}} D_{t}^{\widetilde{\beta}} f\right]$

$$
=q^{-(\widetilde{\alpha}+\widetilde{\beta})} \mathcal{T}[p, q], \quad \hat{S}_{2}\left[D_{t}^{\widetilde{\alpha}} D_{x}^{\widetilde{\beta}} f\right]
$$$$
=q^{-\widetilde{\alpha}} p^{-\widetilde{\beta}} \mathcal{T}[p, q]
$$$$
\hat{S}_{2}\left[D_{x}^{\widetilde{\beta}} D_{t}^{\widetilde{\beta}} f\right]=q^{-\widetilde{\beta}} p^{-\widetilde{\beta}} \mathcal{T}[p, q], \quad \hat{S}_{2}\left[D_{x}^{\widetilde{\beta}} D_{x}^{\widetilde{\beta}} f\right]
$$$$
=p^{-2 \widetilde{\beta}}(\mathcal{T}[p, q]
$$$$
\left.-\Gamma(\tilde{\alpha}+\tilde{\beta}+1) q^{\widetilde{\alpha}+\widetilde{\beta}} p^{\widetilde{\beta}}\right)
$$

Consequently, after operating double Sumudu transform for both sides of (5) and simplify, get

$$
\begin{aligned}
q^{-(\widetilde{\alpha}+\widetilde{\beta}) \hat{\mathcal{T}}[p, q]} & -p^{-\widetilde{\beta}} q^{-\widetilde{\alpha}} \hat{\mathcal{T}}[p, q] \\
& -p^{-\widetilde{\beta}} q^{-\widetilde{\beta}} \widehat{\mathcal{T}}[p, q] \\
& +p^{-2 \widetilde{\beta}}(\widehat{\mathcal{T}}[p, q] \\
& \left.-\Gamma(\tilde{\alpha}+\tilde{\beta}+1) q^{\widetilde{\alpha}+\widetilde{\beta}} p^{\widetilde{\beta}}\right) \\
& =\Gamma(\tilde{\alpha}+\widetilde{\beta}+1)\left(\Gamma(\tilde{\beta}+1) p^{\widetilde{\beta}}\right. \\
& \left.-\Gamma(\tilde{\beta}+1) q^{\widetilde{\beta}}-\Gamma(\tilde{\beta}+1) q^{\widetilde{\alpha}}\right)
\end{aligned}
$$

Thus,

$\left(p^{2 \widetilde{\beta}}-p^{\widetilde{\beta}} q^{\widetilde{\beta}}-p^{\widetilde{\beta}} q^{\widetilde{\alpha}}+q^{\widetilde{\alpha}+\widetilde{\beta}}\right) \mathcal{T}[p, q]=$ $\Gamma(\tilde{\beta}+1) \Gamma(\tilde{\alpha}+\tilde{\beta}+1) q^{2(\widetilde{\alpha}+\widetilde{\beta})} p^{\widetilde{\beta}}+\Gamma(\tilde{\alpha}+\tilde{\beta}+$ 1) $\left(\Gamma(\tilde{\beta}+1) p^{\widetilde{\beta}}-\Gamma(\tilde{\beta}+1) q^{\widetilde{\beta}}-\Gamma(\tilde{\beta}+1) q^{\widetilde{\alpha}}\right)$

$$
\begin{aligned}
\left(p^{2 \widetilde{\beta}}-p^{\widetilde{\beta}} q^{\widetilde{\beta}}-\right. & \left.p^{\widetilde{\beta}} q^{\widetilde{\alpha}}+q^{\widetilde{\alpha}+\widetilde{\beta}}\right) \mathcal{T}[p, q] \\
& =\Gamma(\tilde{\beta}+1) \Gamma(\tilde{\alpha}+\widetilde{\beta} \\
& +1) q^{\widetilde{\alpha}+\widetilde{\beta}} p^{\widetilde{\beta}}\left(p^{2 \widetilde{\beta}}-p^{\widetilde{\beta}} q^{\widetilde{\beta}}\right. \\
& \left.-p^{\widetilde{\beta}} q^{\widetilde{\alpha}}+q^{\widetilde{\alpha}+\widetilde{\beta}}\right)
\end{aligned}
$$

$\widehat{\mathcal{T}}[p, q]=\Gamma(\tilde{\beta}+1) \Gamma(\tilde{\alpha}+\tilde{\beta}+1) q^{\widetilde{\alpha}+\widetilde{\beta}} p^{\widetilde{\beta}}$

By applying the inverse double Sumudu transform of the last equation

$$
f(x, t)=x^{\widetilde{\beta}} t^{\widetilde{\alpha}+\widetilde{\beta}}
$$

Equation (5), has $f(x, t)=x^{2} t^{3}$ as an exact solution when $\tilde{\alpha}=1, \tilde{\beta}=2$.

In the same manner of previous examples, the solution by applying double Elzaki transform can be done for this example to have the same solution of the equation (5)

Some plotting of approximate solutions are given in Fig.4.

Also, Table 5 shows the results of absolutely error for some 10- order approximate solutions.

Table 5. Absolutely error for some approximate solutions of equation (5) for different values of $\tilde{\boldsymbol{\beta}}, \tilde{\boldsymbol{\alpha}}$.

\begin{tabular}{clllll}
\hline $\boldsymbol{( x , t )}$ & \multicolumn{1}{c}{$\boldsymbol{U}_{\text {exact }}$} & \multicolumn{1}{c}{$\boldsymbol{U}_{\text {app }}$} & \multicolumn{1}{c}{$\mid$ Error $\mid$} & \multicolumn{1}{c}{$\boldsymbol{U}_{\text {app } .}$} & $\mid$ Error $\mid$ \\
\hline & $\tilde{\alpha}=\tilde{\beta}=2$ & $\tilde{\alpha}=\mathbf{1 . 6}, \tilde{\beta}=\mathbf{1 . 3}$ & & $\tilde{\alpha}=\mathbf{1 . 8}, \tilde{\beta}=\mathbf{1 . 2}$ & \\
$(0.3,0.1)$ & $9 \times 10^{-6}$ & $2.6318287761 \mathrm{e}-6$ & $2.5418287761 \mathrm{e}-4$ & 0.0002358009 & $2.2680092568 \mathrm{e}-4$ \\
$(0.6,0.2)$ & $5.76 \times 10^{-6}$ & $4.8370838172 \mathrm{e}-3$ & $4.2610838172 \mathrm{e}-3$ & 0.0043338262 & $3.7578261669 \mathrm{e}-3$ \\
$(0.40 .8)$ & 0.065536 & 0.1590885601 & $9.355256007 \mathrm{e}-2$ & 0.1705068969 & $1.0497089688 \mathrm{e}-1$ \\
$(0.9,0.9)$ & 0.531441 & 0.6424192405 & $1.1097824055 \mathrm{e}-1$ & 0.6424192405 & $1.1097824055 \mathrm{e}-1$ \\
$(0.7,0.3)$ & 0.003969 & 0.0191548648 & $1.5185864788 \mathrm{e}-2$ & 0.0175987334 & $1.3629733395 \mathrm{e}-2$ \\
$(0.4,0.3)$ & 0.001296 & 0.0092540028 & $7.958002818 \mathrm{e}-3$ & 0.0089915746 & $7.6955746399 \mathrm{e}-3$ \\
$(0.6,0.7)$ & 0.086436 & 0.1829704438 & $9.6534443829 \mathrm{e}-2$ & 0.1858127969 & $9.9376796908 \mathrm{e}-2$ \\
$(0.2,0.6)$ & 0.005184 & 0.0280528908 & $2.2868890756 \mathrm{e}-2$ & 0.0313104815 & $2.6126481471 \mathrm{e}-2$ \\
\hline
\end{tabular}



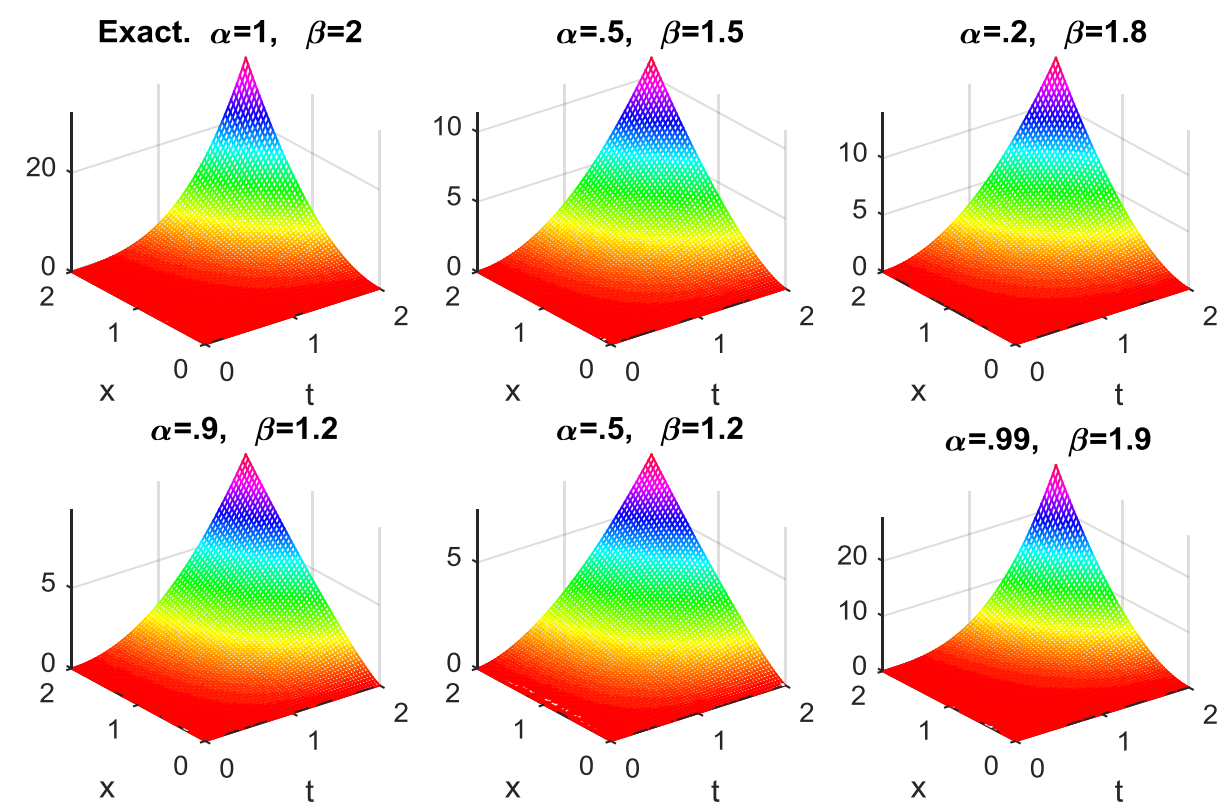

Figure 4. Numerical solution with different values of $\tilde{\boldsymbol{\beta}} \tilde{\boldsymbol{\alpha}}$. of equation (5) converge to the exact solution.

\section{Discussion:}

In this paper used the double Sumudu and Elzaki transformations to compute some of fractional partial differential equations which are proposed as a new approach in this research for these equations to show that the two transformations efficient and accurate by applying these methods and showing perfect results in numerical tables and graphics of the illustrative numerical examples from the increasing of the fractional order convergent to the numerical solution in positive integer through the absolute error goes to zero.

The Sumudu transformation may be used to solve problem without resorting to a new frequency domain and having scale and unit-preserving properties. The Sumudu transform is used to solve PDE by transforming to algebraic equation and after using some mathematical algebra technique using inverse Sumudu transformation to get up the solution. Also the Elzaki transformation is used when the Sumudu transformation cannot solve some of fractional ordinary differential equation when the coefficients are variable, on the other hand the Elzaki transform only can solve such of such equations with changes of the initial conditions of the problem, this is the only advantage of Elzaki transform over the Sumudu one.

Usually, both Sumudu and Elzaki transforms are used to solve some of FPDE with constant coefficients to get the analytic solution. So in this case, need not to study the stability or convergence of the solution and the tables and figures explain the numerical values of the solution with different values of $\tilde{\alpha}$ and $\tilde{\beta}$.

\section{Conclusion:}

In this paper, introduced the double Sumudu and double Elzaki transforms to find the solution for several types of linear partial differential equations with constant coefficients that include space, time and space-time or mixed fractional Caputo derivatives, in this case both transforms give the same solution. All problems which are argued proved the efficiency of this methods throughout the plotting and numerical calculus of approximated solutions.

\section{Authors' declaration:}

- Conflicts of Interest: None.

- We hereby confirm that all the Figures and Tables in the manuscript are mine ours. Besides, the Figures and images, which are not mine ours, have been given the permission for republication attached with the manuscript.

- Ethical Clearance: The project was approved by the local ethical committee in University of Baghdad.

\section{References:}

1. Li Z, Liu L, Dehghan S, Chen Y, Xue DA. Review and evaluation of numerical tools for fractional calculus and fractional order controls. Int $\mathbf{J}$ control. 2017; 90(6): 1165-1181.

2. Jarad F, Tas K. Application of Sumudu and double Sumudu transforms to Caputo-fractional differential 
equations, J Comput Anal Appl. 2012;14(3): 475 483.

3. Eslaminasab M, Abbasbandy S. Study on usage of Elzaki transform for the ordinary differential equations with non-constant coefficients. IJIM 2015; 7(3): $277-282$.

4. Tarik M, Salih M. On the Elzaki transform and ordinary differential equation with variable coefficients. Adv Theor App Math. 2011; 6(1): 41 46.

5. Tarik M, Salih M, Eman M. Elzaki and Sumudu transform for solving some differential equations. Glob. J. Pure Appl. Math. 2012; 8(2): 167 - 173.

6. Nuruddeen R, Lawal M, Nass A, Sulaimman T. A Review of the integral transforms-based decomposition methods and their applications in solving nonlinear partial differential equations. J Math. 2018; 7(1): 262-280.

7. Eltayeb H , Kiliçman A. On double Sumudu transform and double Laplace transform. Malayas J Math Sci. 2010; 4(1): 17-30.

8. Mehdi D. Application of fractional Calculus. J Math C Sc. 2010; 4(21):1021 - 1032.

9. Jun J, Yuqiang F, Shougui L. Exact solutions to the fractional differential equations with mixed partial derivatives. J Axioms. 2018; 7(10): 1 - 18.
10. Banu Y. Generalized Mittag-Leffler function and its properties. Ntmsci. 2015; 3(1): $12-18$.

11. Rakash K. A class of extended Mittag-Leffler functions and their properties related to integral transforms and fractional calculus. J Math. 2015; 9(3):1069 - 1082 .

12. Kilicman A , Gadian H. On the applications of Laplace and Sumudu transforms. J Franklin I. 2010; 3(7). $848-862$.

13. HawJoon K. The time shifting theorem and the convolution for Elzaki transform. Int J App Math. 2013; 87(2):261 - 271.

14. Abaker A, Yagoub A. On double Elzaki transform and double Laplace transform. J Math. 2015; 11(1): $35-41$.

15. Undegaonkar H. Similarity and comparison of Sumudu transform with Elzaki transform in solving ordinary differential equations with variable coefficients. Int J Math. 2017; 8(3): $66-69$.

16. Fatima A, Eltayeb A, Arbab I. A new technique of Laplace variational iteration method for solving space-time fractional telegraph equations. Int $\mathbf{J}$ Diff Eq. 2013; 2(13): $1-10$.

\footnotetext{
حل المعادلات التفاضلية الجزئية الكسرية باستخدام التحويلات الخطية المضاعفة سمير قاسم حسن 2 حسن شذر كاظم 1 1قسم الرياضيات، كلية التربية، جامعة بغداد، بغداد، العراق.

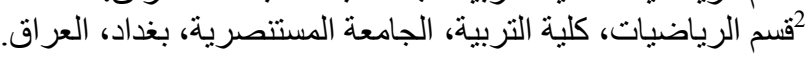

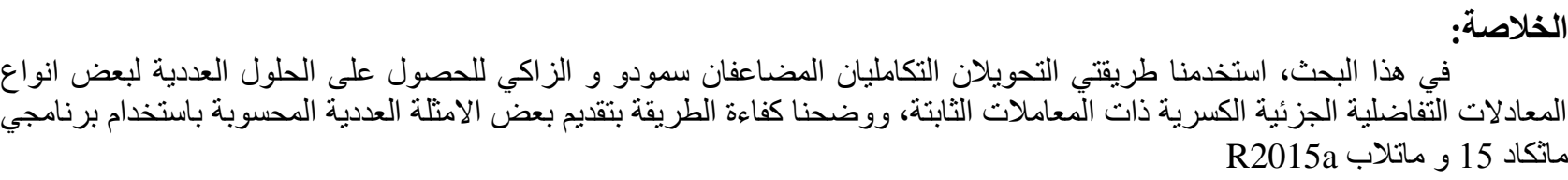
الكلمات المفتاحية: مشتقة كابونا، التفاضل الكسري، المعادلات التفاضلية الجزئية الكسرية، تحويل سمودو المفرد و المضاعف، تحويل الز اكي.
} 